\title{
Measurement of the strong coupling constant $\alpha_{\mathrm{s}}$ for bottom quarks at the $Z^{0}$ resonance
}

\section{L3 Collaboration}

B. Adeva ${ }^{a}$, O. Adriani ${ }^{\text {b }}$, M. Aguilar-Benitez ${ }^{\text {c }}$, H. Akbari ${ }^{\text {d }}$, J. Alcaraz ${ }^{c}$, A. Aloisio ${ }^{\mathrm{e}}$, G. Alverson ${ }^{\text {, }}$, M.G. Alviggi e, G. Ambrosi ${ }^{\mathrm{g}}$, Q. An ${ }^{\mathrm{h}}$, H. Anderhub ${ }^{\mathrm{i}}$, A.L. Anderson ${ }^{\mathrm{j}}$, V.P. Andreev ${ }^{k}$, T. Angelov ${ }^{j}$, L. Antonov ${ }^{\ell}$, D. Antreasyan ${ }^{m}$, P. Arce ${ }^{c}$, A. Arefiev ${ }^{n}$, T. Azemoon ${ }^{\circ}$, T. Aziz ${ }^{\text {}}$, P.V.K.S. Baba ${ }^{\text {h }}$, P. Bagnaia ${ }^{\mathrm{q}}$, J.A. Bakken ${ }^{\mathrm{r}}$, L. Baksay ${ }^{\text {s }}$, R.C. Ball ${ }^{\circ}$, S. Banerjee ${ }^{p}$, J. Bao $^{d}$, R. Barillère ${ }^{a}$, L. Barone ${ }^{q}$, R. Battiston ${ }^{\text {g }}$, A. Bay ${ }^{\text {t }}$, U. Becker ${ }^{j}$,

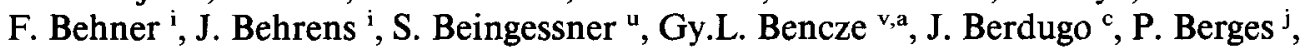
B. Bertucci ${ }^{\text {g }}$, B.L. Betev ${ }^{\ell}$, A. Biland ${ }^{\text {i }}$, G.M. Bilei ${ }^{\text {g }}$, R. Bizzarri ${ }^{\mathrm{q}}$, J.J. Blaising ${ }^{4}$, P. Blömeke ${ }^{\text {w }}$, B. Blumenfeld ${ }^{\text {d }}$, G.J. Bobbink ${ }^{x}$, M. Bocciolini ${ }^{\text {b }}$, R. Bock ${ }^{w}$, A. Böhm ${ }^{\text {w,a }}$, B. Borgia ${ }^{\mathrm{a}}$, D. Bourilkov ${ }^{\ell}$, M. Bourquin ${ }^{t}$, D. Boutigny ${ }^{u}$, B. Bouwens ${ }^{\text {x }}$, E. Brambilla ${ }^{\mathrm{e}}$, J.G. Branson ${ }^{\mathrm{y}}$, I.C. Brock ${ }^{z}$, F. Bruyant ${ }^{a}$, C. Buisson ${ }^{\text {aa }}$, A. Bujak ${ }^{\text {ab }}$, J.D. Burger ${ }^{j}$, J.P. Burq ${ }^{\text {aa }}$, J. Busenitz ${ }^{\text {s }}$, X.D. Cai ${ }^{\text {h}}$, M. Capell ${ }^{\text {ac }}$, M. Caria ${ }^{\mathrm{g}}$, G. Carlino ${ }^{\mathrm{e}}$, F. Carminati ${ }^{\mathrm{b}}$, A.M. Cartacci ${ }^{\mathrm{b}}$, M. Cerrada ${ }^{\mathrm{c}}$, F. Cesaroni ${ }^{\text {q }}$, Y.H. Chang ${ }^{j}$, U.K. Chaturvedi ${ }^{\text {h}}$, M. Chemarin ${ }^{\text {aa }}$, A. Chen ${ }^{\text {ad }}$, C. Chen ${ }^{\text {ae }}$, G.M. Chen ${ }^{\text {ac }}$, H.F. Chen af , H.S. Chen ${ }^{\text {ae }}$, M. Chen ${ }^{\text {j, M.L. Chen }}{ }^{\circ}$, W.Y. Chen ${ }^{\text {h }}$, G. Chiefari ${ }^{\text {e }}$, C.Y. Chien ${ }^{d}$, M. Chmeissani ${ }^{\circ}$, C. Civinini ${ }^{b}$, I. Clare ${ }^{j}$, R. Clare ${ }^{j}$, H.O. Cohn ${ }^{\text {ag }}$, G. Coignet ${ }^{\text {u, }}$ N. Colino ${ }^{\text {a }}$, V. Commichau ${ }^{\text {w }}$, G. Conforto ${ }^{b}$, A. Contin ${ }^{\text {m,a }}$, F. Crijns ${ }^{\text {ah }}$, X.Y. Cui ${ }^{\text {h }}$, T.S. Dai ${ }^{j}$, R. D'Alessandro ${ }^{b}$, R. de Asmundis ${ }^{e}, A$. Degré ${ }^{\mathrm{a}, \mathrm{u}}, \mathrm{K}$. Deiters ${ }^{\mathrm{j}}, \mathrm{E}$. Dénes ${ }^{\mathrm{v}, \mathrm{a}}, \mathrm{P}$. Denes ${ }^{\mathrm{r}}$, F. DeNotaristefani ${ }^{\text {q }}$, M. Dhina ${ }^{i}$, D. DiBitonto ${ }^{\text {s }}$, M. Diemoz ${ }^{\mathrm{q}}$, H.R. Dimitrov ${ }^{\ell}$, C. Dionisi ${ }^{\mathrm{q}}$, M.T. Dova ${ }^{\text {h}}$, E. Drago ${ }^{\mathrm{e}}$, T. Driever ${ }^{\text {ah }}$, D. Duchesneau ${ }^{\mathrm{t}}$, P. Duinker ${ }^{\mathrm{x}}$, I. Duran ${ }^{\mathrm{c}}$, H. El Mamouni ${ }^{\text {aa }}$, A. Engler ${ }^{z}$, F.J. Eppling ${ }^{j}$, F.C. Erné ${ }^{x}$, P. Extermann ${ }^{t}$, R. Fabbretti ${ }^{\text {ai }}$, M. Fabre ${ }^{\text {i }}$, S. Falciano ${ }^{\text {q }}$, Q. Fan ${ }^{\text {h }}$, S.J. Fan ${ }^{\text {aj }}$, O. Fackler ${ }^{\text {ac }}$, J. Fay ${ }^{\text {aa }}$, T. Ferguson ${ }^{\text {z }}$, G. Fernandez ${ }^{\text {c }}$, F. Ferroni ${ }^{\text {a,a }}$, H. Fesefeldt ${ }^{\text {w }}$, E. Fiandrini ${ }^{\text {g }}$, J. Field ${ }^{\text {' }}$, F. Filthaut ${ }^{\text {ah }}$, G. Finocchiaro ${ }^{\mathrm{q}}$, P.H. Fisher ${ }^{\mathrm{d}}$, G. Forconi ${ }^{\mathrm{t}}$, T. Foreman ${ }^{\mathrm{x}}$, K. Freudenreich ${ }^{\mathrm{i}}$, W. Friebel ${ }^{\text {ak }}$, M. Fukushima ${ }^{j}$, M. Gailloud ${ }^{a \ell}$, Yu. Galaktionov ${ }^{n}$, E. Gallo ${ }^{b}$, S.N. Ganguli ${ }^{\text {p }}$, P. Garcia-Abia ${ }^{c}$, S.S. Gau ${ }^{\text {ad }}$, D. Gele ${ }^{\text {aa }}$, S. Gentile ${ }^{\mathrm{q}}$, M. Glaubman ${ }^{\mathrm{f}}$, S. Goldfarb ${ }^{\circ}$, Z.F. Gong ${ }^{\text {af }}$, E. Gonzalez ${ }^{\mathrm{c}}$,

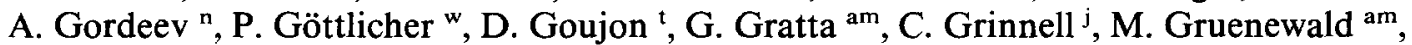
M. Guanziroli ${ }^{\text {h }}$, J.K. Guo aj, A. Gurtu ${ }^{\text {p }}$, H.R. Gustafson ${ }^{\circ}$, L.J. Gutay ${ }^{\text {ab }}$, H. Haan ${ }^{\text {w }}$, A. Hasan ${ }^{\text {h }}$, D. Hauschildt ${ }^{x}$, C.F. He ${ }^{\text {aj }}$, T. Hebbeker ${ }^{w}$, M. Hebert ${ }^{y}$, G. Herten ${ }^{j}$, U. Herten ${ }^{\text {w }}$, A. Hervé a , K. Hilgers ${ }^{\text {w }}$, H. Hofer ${ }^{\mathrm{i}}$, H. Hoorani ${ }^{\text {h }}$, L.S. Hsu ${ }^{\text {ad }}$, G. Hu ${ }^{\text {h }}$, G.Q. Hu ${ }^{\text {aj }}$, B. Ille ${ }^{\text {aa }}$, M.M. Ilyas ${ }^{\mathrm{h}}$, V. Innocente ${ }^{\mathrm{e}, \mathrm{a}}$, H. Janssen ${ }^{\mathrm{a}}$, S. Jezequel ${ }^{\mathrm{u}}$, B.N. Jin ${ }^{\mathrm{a}}$, L.W. Jones ${ }^{\circ}$, A. Kasser ${ }^{\mathrm{a} \ell}$, R.A. Khan ${ }^{\text {h }}$, Yu. Kamyshkov n,ag, Y. Karyotakis ${ }^{\text {u,a }}$, M. Kaur ${ }^{\text {h }}$, S. Khokhar ${ }^{\text {h }}$, V. Khoze ${ }^{\text {, }}$, M.N. Kienzle-Focacci ${ }^{\text {t }}$, W. Kinnison ${ }^{\text {an }}$, D. Kirkby ${ }^{\text {am }}$, W. Kittel ${ }^{\text {ah }}$, A. Klimentov ${ }^{\text {, }}$,

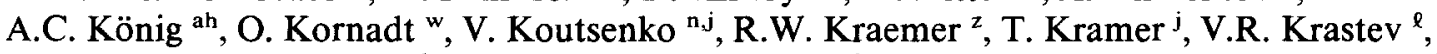
W. Krenz ${ }^{\text {w }}$, J. Krizmanic ${ }^{\text {d }}$, K.S. Kumar ${ }^{\text {ao }}$, V. Kumar ${ }^{\text {h }}$, A. Kunin ${ }^{\text {ao,n }}$, V. Lalieu ${ }^{\text {t }}$, G. Landi ${ }^{b}$, K. Lanius ${ }^{\text {a }}$, D. Lanske ${ }^{\text {w }}$, S. Lanzano ${ }^{\text {e }}$, P. Lebrun ${ }^{\text {aa }}$, P. Lecomte ${ }^{\text {i }}$, P. Lecoq ${ }^{\text {a }}$, P. Le Coultre ${ }^{\mathrm{i}}$, D. Lee $^{\text {an }}$, I. Leedom ${ }^{f}$, J.M. Le Goff ${ }^{a}$, L. Leistam $^{a}$, R. Leiste ${ }^{\text {ak }}$, M. Lenti ${ }^{b}$, E. Leonardi ${ }^{\mathrm{a}}$, J. Lettry ${ }^{i}$, P.M. Levchenko ${ }^{k}$, X. Leytens ${ }^{x}$, C. Li ${ }^{\text {af,h }}$, H.T. Li ${ }^{\text {ae }}$, J.F. Li ${ }^{\text {h }}$, L. Li $^{\text {ai }}$, P.J. Li aj, Q. Li ${ }^{\text {}}$, X.G. Li ${ }^{\text {ae }}$, J.Y. Liao aj, Z.Y. Lin af , F.L. Linde ${ }^{a, x}$, B. Lindemann ${ }^{\text {w }}$, D. Linnhofer ${ }^{i}$, R. Liu ${ }^{\text {h }}$, Y. Liu ${ }^{\text {h }}$, W. Lohmann ${ }^{\text {ak }}$, E. Longo ${ }^{\text {a }}$, Y.S. Lu ${ }^{\text {ae }}$, J.M. Lubbers ${ }^{\text {a }}$, K. Lübelsmeyer ${ }^{\text {w }}$, 
C. Luci ${ }^{a}$, D. Luckey ${ }^{\mathrm{m}, \mathrm{j}}$, L. Ludovici ${ }^{\mathrm{q}}$, L. Luminari ${ }^{\text {, }}$, W.G. Ma ${ }^{\text {af }}$, M. MacDermott ${ }^{\mathrm{i}}$, R. Magahiz ${ }^{\text {ap }}$, P.K. Malhotra ${ }^{\text {p }}$, R. Malik ${ }^{\text {h }}$, A. Malinin ${ }^{\text {n,u }}$, C. Maña ${ }^{\text {c }}$, D.N. Mao ${ }^{\circ}$, Y.F. Mao ${ }^{\text {ae }}$, M. Maolinbay i, P. Marchesini i, A. Marchionni b, J.P. Martin ${ }^{\text {aa }}$, L. Martinez-Laso ${ }^{\text {a }}$, F. Marzano ${ }^{q}$, G.G.G. Massaro ${ }^{\text {, }}$, T. Matsuda ${ }^{j}$, K. Mazumdar ${ }^{\text {p }}$, P. McBride ${ }^{\text {ao }}$, T. McMahon $^{\text {ab }}$, D. McNally ${ }^{i}$, Th. Meinholz ${ }^{\text {w }}$, M. Merk ${ }^{\text {ah }}$, L. Merola ${ }^{\text {e }}$, M. Meschini ${ }^{\text {b }}$, W.J. Metzger ${ }^{\text {ah }}$, Y. Mi $^{\text {h }}$, G.B. Mills an, Y. Mir ${ }^{\text {h }}$, G. Mirabelli a, J. Mnich ${ }^{\text {w }}$, M. Möller ${ }^{\text {w }}$, B. Monteleoni ${ }^{\text {b }}$, G. Morand ", R. Morand ", S. Morganti ${ }^{\text {q, N.E. Moulai }}{ }^{\text {h }}$, R. Mount ${ }^{\text {am }}$, S. Müller ${ }^{\text {w }}$, E. Nagy v, M. Napolitano ${ }^{\text {e }}$, H. Newman ${ }^{\text {am }}$, C. Neyer ${ }^{i}$, M.A. Niaz ${ }^{\text {h }}$, L. Niessen ${ }^{\text {w }}$, H. Nowak ${ }^{\text {ak }}$, D. Pandoulas ${ }^{\text {w }}$, M. Pauluzzi ${ }^{\mathrm{g}}$, F. Pauss ${ }^{\text {i }}$, F. Plasil ag, G. Passaleva ${ }^{\mathrm{b}}$, S. Patricelli e, Y.J. Pei w, D. Perret-Gallix ${ }^{\text {, }}$, J. Perrier ${ }^{\text {, }}$, A. Pevsner ${ }^{\text {d }}$, M. Pieri ${ }^{\text {b }}$, P.A. Piroué ${ }^{r}$, V. Plyaskin ${ }^{\text {n }}$, M. Pohl i ${ }^{\text {, }}$ V. Pojidaev ${ }^{\text {n }}$, N. Produit ${ }^{\text {t }}$ J.M. Qian ${ }^{\circ}$, K.N. Qureshi ${ }^{\text {h }}$, R. Raghavan ${ }^{\text {p }}$, G. Rahal-Callot ${ }^{\text {, }}$, G. Raven ${ }^{\text {x }}$ P. Razis ${ }^{\text {s }}$, K. Read ${ }^{\text {ag }}$, D. Ren ${ }^{i}$, Z. Ren ${ }^{\text {h }}$, S. Reucroft ${ }^{f}$, A. Ricker ${ }^{\text {w }}$, S. Riemann ${ }^{\text {ak }}$, O. Rind ${ }^{\circ}$, C. Rippich ${ }^{z}$, H.A. Rizvi ${ }^{\text {h }}$, B.P. Roe ${ }^{\circ}$, M. Röhner ${ }^{w}$, S. Röhner ${ }^{\text {w }}$, L. Romero ${ }^{\text {, }}$, J. Rose ${ }^{\text {w }}$, S. Rosier-Lees ${ }^{\text {u }}$, R. Rosmalen ${ }^{\text {ah }}$, Ph. Rosselet ${ }^{\text {al }}$, A. Rubbia ${ }^{\mathrm{j}}$, J.A. Rubio ${ }^{\text {a,c }}$, W. Ruckstuhl t ${ }^{\mathrm{t}}$ H. Rykaczewski ${ }^{\mathrm{i}}$, M. Sachwitz ${ }^{\text {ak,a }}$, J. Salicio ${ }^{\text {a.c }}$, J.M. Salicio ${ }^{\mathrm{c}}$, G. Sanders ${ }^{\text {an }}$, A. Santocchia ${ }^{\text {g }}$, M.S. Sarakinos ${ }^{\text {j, G. Sartorelli }}{ }^{\mathrm{m}, \mathrm{h}}$, G. Sauvage ", A. Savin ${ }^{\mathrm{n}}$, V. Schegelsky k, K. Schmiemann ${ }^{\text {w }}$, D. Schmitz w, P. Schmitz w, M. Schneegans ", H. Schopper aq, D.J. Schotanus ${ }^{\text {ah }}$, S. Shotkin ${ }^{j}$, H.J. Schreiber ${ }^{\text {ak }}$, R. Schulte ${ }^{\text {w }}$, S. Schulte ${ }^{\text {w }}$, K. Schultze ${ }^{\text {w }}$, J. Schütte ${ }^{\text {ao }}$, J. Schwenke ${ }^{\text {w }}$, G. Schwering ${ }^{\text {w }}$, C. Sciacca ${ }^{\text {e }}$, I. Scott ${ }^{\text {ao }}$, R. Sehgal ${ }^{\text {h}}$, P.G. Seiler ${ }^{\text {ai }}$, J.C. Sens ${ }^{x}$, L. Servoli ${ }^{g}$, I. Sheer ${ }^{y}$, D.Z. Shen ${ }^{\text {aj }}$, V. Shevchenko ${ }^{n}$, S. Shevchenko ${ }^{n}$, X.R. Shi ${ }^{\text {am }}$, K. Shmakov ${ }^{n}$, V. Shoutko ${ }^{n}$, E. Shumilov ${ }^{n}$, N. Smirnov ${ }^{\text {k }}$, E. Soderstrom ${ }^{r}$, A. Sopczak ${ }^{y}$, C. Spartiotis ${ }^{d}$, T. Spickermann ${ }^{w}$, P. Spillantini ${ }^{b}$, R. Starosta ${ }^{w}$, M. Steuer ${ }^{\text {m,j }}$, D.P. Stickland ${ }^{r}$, F. Sticozzi ${ }^{j}$, W. Stoeffl ${ }^{\text {ac }}$, H. Stone ${ }^{\mathfrak{t}}$, K. Strauch ${ }^{\text {ao }}$, B.C. Stringfellow ${ }^{\text {ab }}$, K. Sudhakar ${ }^{p, w}$, G. Sultanov ${ }^{\text {h }}$, R.L. Sumner ${ }^{\text {r }}$, L.Z. Sun ${ }^{\text {af,h }}$, H. Suter ${ }^{\text {i }}$, R.B. Sutton ${ }^{\text {z }}$, J.D. Swain ${ }^{\text {h }}$, A.A. Syed ${ }^{\text {h }}$, X.W. Tang ae, E. Tarkovsky ${ }^{n}$, L. Taylor ${ }^{f}$, C. Timmermans ${ }^{\text {ah }}$, Samuel C.C. Ting ${ }^{j}$, S.M. Ting ${ }^{j}$, Y.P. Tong ${ }^{\text {ad }}$, F. Tonisch ${ }^{\text {ak }}$, M. Tonutti ${ }^{\text {w }}$, S.C. Tonwar ${ }^{\mathrm{p}}$, J. Tóth ${ }^{\mathrm{v}, \mathrm{a}}$, G. Trowitzsch $^{\text {ak}}$, C. Tully ${ }^{a m}$, K.L. Tung ${ }^{\text {ae }}$, J. Ulbricht ${ }^{i}$, L. Urbán ${ }^{\mathrm{v}}$, U. Uwer ${ }^{\text {w }}$, E. Valente ${ }^{\mathrm{q}}$,

R.T. Van de Walle ${ }^{\text {ah }}$, I. Vetlitsky ${ }^{n}$, G. Viertel ${ }^{\mathrm{i}}$, P. Vikas ${ }^{\mathrm{h}}$, U. Vikas ${ }^{\mathrm{h}}$, M. Vivargent ${ }^{\mathrm{u}, \mathrm{j}}$, H. Vogel ${ }^{\text {z }}$, H. Vogt $^{\text {ak }}$, G. Von Dardel a , I. Vorobiev ${ }^{n}$, A.A. Vorobyov ${ }^{k}$, An.A. Vorobyov ${ }^{k}$, L. Vuilleumier ${ }^{\text {al }}$, M. Wadhwa ${ }^{\mathrm{h}}$, W. Wallraff ${ }^{\text {w }}$, C.R. Wang ${ }^{\text {af }}$, G.H. Wang ${ }^{\text {, }}$, J.H. Wang ${ }^{\text {ae }}$, Q.F. Wang ${ }^{\text {ao }}$, X.L. Wang af, Y.F. Wang ${ }^{b}$, Z. Wang ${ }^{\text {h }}$, Z.M. Wang ${ }^{\text {h,af }}$, A. Weber ${ }^{\text {w }}$, J. Weber ${ }^{\text {i }}$, R. Weill ${ }^{a \ell}$, T.J. Wenaus ${ }^{\text {ac }}$, J. Wenninger $^{\mathrm{t}}$, M. White ${ }^{\mathrm{j}}, \mathrm{C}$. Willmott ${ }^{\mathrm{c}}, \mathrm{F}$. Wittgenstein ${ }^{\mathrm{a}}$, D. Wright ${ }^{r}$, R.J. Wu ${ }^{\text {ae }}$, S.L. Wu ${ }^{\text {h }}$, S.X. Wu ${ }^{\text {h }}$, Y.G. Wu ${ }^{\text {ae }}$, B. Wystouch ${ }^{j}$, Y.Y. Xie ${ }^{\text {aj }}$,

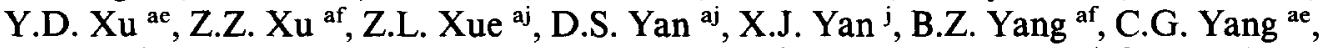
G. Yang ${ }^{\text {h }}$, K.S. Yang ${ }^{\text {ae }}$, Q.Y. Yang ${ }^{\text {ae }}$, Z.Q. Yang ${ }^{\text {aj }}$,C.H. Ye ${ }^{\text {h }}$, J.B. Ye ${ }^{\text {i,af }}$, Q. Ye ${ }^{\text {h }}$, S.C. Yeh ad, Z.W. Yin ${ }^{\text {aj }}$, J.M. You ${ }^{\text {h }}$, M. Yzerman ${ }^{x}$, C. Zaccardelli am , P. Zemp ${ }^{\text {i }}$, M. Zeng ${ }^{\text {h }}$, Y. Zeng ${ }^{\text {w }}$, D.H. Zhang ${ }^{x}$, Z.P. Zhang af,h , J.F. Zhou ${ }^{\text {w }}$, R.Y. Zhu ${ }^{\text {am }}$, H.L. Zhuang ${ }^{\text {ae }}$ and A. Zichichi ${ }^{\text {m,a,h }}$

a European Laboratory for Particle Physics, CERN, CH-1211 Geneva 23, Switzerland

b INFN - Sezione di Firenze and University of Florence, I-50125 Florence, Italy

c Centro de Investigaciones Energeticas, Medioambientales y Tecnologicas, CIEMAT, E-28040 Madrid, Spain

d Johns Hopkins University, Baltimore, MD 21218, USA

- INFN - Sezione di Napoli and University of Naples, I-80125 Naples, Italy

f Northeastern University, Boston, MA 02115, USA

I INFN - Sezione di Perugia and Universitá Degli Studi di Perugia, I-06100 Perugia, Italy

h World Laboratory, FBLJA Project, CH-1211 Geneva, Switzerland

Eidgenössische Technische Hochschule, ETH Zürich, CH-8093 Zurich, Switzerland

j Massachusetts Institute of Technology, Cambridge, MA 02139, USA

k Leningrad Nuclear Physics Institute, SU-188 350 Gatchina, USSR

$\&$ Central Laboratory of Automation and Instrumentation, CLANP, Sofia, Bulgaria

m INFN - Sezione di Bologna, I-40126 Bologna, Italy 
n Institute of Theoretical and Experimental Physics, ITEP, SU-117259 Moscow, USSR

- University of Michigan, Ann Arbor, MI 48109, USA

- Tata Institute of Fundamental Research, Bombay 400005 , India

" INFN - Sezione di Roma and University of Rome "La Sapienza", I-00185 Rome, Italy

r Princeton University, Princeton, NJ 08544, USA

s University of Alabama, Tuscaloosa, AL 35486, USA

- University of Geneva, CH-1211 Geneva 4, Switzerland

" Laboratoire de Physique des Particules, LAPP, F-74519 Annecy-le-Vieux, France

$\checkmark$ Central Research Institute for Physics of the Hungarian Academy of Sciences, H-1525 Budapest 114, Hungary

" I. Physikalisches Institut, RWTH, W-5100 Aachen, FRG' and III. Physikalisches Institut, RWTH, W-5100 Aachen, FRG ${ }^{1}$

× National Institute for High Energy Physics, NIKHEF, NL-1009 DB Amsterdam, The Netherlands

y University of California, San Diego, CA 92182, USA

2 Carnegie Mellon University, Pittsburgh, PA 15213, USA

as Institut de Physique Nucléaire de Lyon, IN2P3-CNRS/Université Claude Bernard, F-69622 Villeurbanne Cedex, France

ab Purdue University, West Lafayette, IN 47907, USA

ac Lawrence Livermore National Laboratory, Livermore, CA 94550, USA

ad High Energy Physics Group, Taiwan, ROC

ae Institute of High Energy Physics, IHEP, Beijing, China

af University of Science and Technology of China, Hefei, Anhui 230 029, China

as Oak Ridge National Laboratory, Oak Ridge, TN 37830, USA

ah University of Nijmegen and NIKHEF, NL-6525 ED Nijmegen, The Netherlands

ai Paul Scherrer Institut (PSI), Würenlingen, Switzerland

aj Shanghai Institute of Ceramics, SIC, Shanghai, China

ak Institut für Hochenergiephysik, O-1615 Zeuthen-Berlin, FRG ${ }^{1}$

al University of Lausanne, CH-1015 Lausanne, Switzerland

am California Institute of Technology, Pasadena, CA 91125, USA

an Los Alamos National Laboratory, Los Alamos, NM 87544, USA

ao Harvard University, Cambridge, MA 02139, USA

ap Union College, Schenectady, NY 12308, USA

aq University of Hamburg, W-2000 Hamburg, FRG

Received 20 August 1991

We have measured the ratio of the strong coupling constants $\alpha_{s}$ for bottom quarks and light quarks at the $Z^{0}$ resonance, in order to test the flavour independence of the strong interaction. The coupling strength $\alpha_{\mathrm{s}}$ has been determined from the fraction of events with three jets, measured for a sample of all hadronic events, and for inclusive muon and electron events. The $b$ purity is evaluated to be $22 \%$ for the first data set and $87 \%$ for the inclusive lepton sample. We find $\alpha_{\mathrm{s}}(\mathrm{b}) / \alpha_{\mathrm{s}}(\mathrm{udsc})=1.00 \pm$ 0.05 (stat.) \pm 0.06 (syst.).

\section{Introduction}

Quantum chromodynamics, the theory of strong interactions, predicts the coupling constant $\alpha_{\mathrm{s}}$ to be independent of the quark flavour.

For the light quark species, up, down and strange, this prediction is supported by the approximate isospin and SU(3) flavour symmetries. The relative coupling strengths for charm and bottom quarks have

1 Supported by the German Bundesministerium für Forschung und Technologie. been measured in $\mathrm{e}^{+} \mathrm{e}^{-}$annihilation at center of mass energies around $30 \mathrm{GeV}[1,2]$, confirming the flavour independence of $\alpha_{\mathrm{s}}$ within large uncertainties. Also the comparison of $\alpha_{\mathrm{s}}$ values measured in charmonium and bottomonium decays [3] with those obtained for other processes involving only $u, d$ and $s$ quarks supports the flavour independence of the strong interaction. However, since these comparisons involve different energy scales and different systematic errors, the accuracy of such tests is limited. The flavour composition in hadronic events produced in $\mathrm{e}^{+} \mathrm{e}^{-}$collisions at the $\mathrm{Z}^{0}$ pole is different 
from that at lower center of mass energies. By comparing the corresponding 3-jet fractions one finds that the strong coupling constants for quarks with different electric charge ( $u, c$ and d, s, b) agree within $10 \%$ [4]. Here we present a precise measurement of the relative coupling strength for bottom quarks at the $Z^{0}$ resonance using the L3 detector at LEP.

The fraction of $b$ events which we measured in hadronic decays of the $Z^{0}$ boson is in agreement with the standard model prediction of $22 \%$ [ 5,6$]$. Bottom quarks can be tagged via their semileptonic decays $b \rightarrow \ell+X$ : because of the large mass of the $b$ quark, the leptons have large momentum $p$ and large transverse momentum $p_{\perp}$ with respect to the nearest jet. By selecting hadronic events containing electrons and muons with large values of $p$ and $p_{\perp}$ we obtain a sample of events with a high $b$ purity.

As the 3-jet rate $\left(\sigma_{3 \text {-jet }} / \sigma_{\text {tot }}\right)$ is a measure of $\alpha_{\mathrm{s}}$, the comparison of the 3-jet fractions for event samples with different flavour composition provides a test of flavour independence. In this letter we determine the ratio of the 3-jet fraction for inclusive lepton events and of the 3-jet fraction for a sample of all hadronic events. In this double ratio, which is sensitive to the ratio of the coupling constants $\alpha_{\mathrm{s}}$ (b)/ $/ \alpha_{\mathrm{s}}$ (udsc), most systematic uncertainties cancel.

The analysis is based on the events collected in 1990 ( $\simeq 5.5 \mathrm{pb}^{-1}$ of integrated luminosity) at center of mass energies in the range $88.2<\sqrt{s}<94.2 \mathrm{GeV}$.

\section{The $\mathrm{L3}$ detector}

The $\mathrm{L} 3$ detector covers $99 \%$ of $4 \pi$. The detector includes a central vertex chamber, a precise electromagnetic calorimeter composed of BGO crystals, a ring of scintillation counters, a uranium and brass hadron calorimeter with proportional wire chamber readout, and a high accuracy muon chamber system. These detectors are installed in a magnet with an inner diameter of $12 \mathrm{~m}$, which provides a uniform field of $0.5 \mathrm{~T}$ along the beam direction.

The central tracking chamber is a time expansion chamber consisting of two cylindrical layers of 12 and 24 sectors, with 62 wires measuring the $R-\phi$ coordinate. The single wire resolution is $58 \mu \mathrm{m}$ averaged over the entire cell. The double track resolution is 640 $\mu \mathrm{m}$. The fine segmentation of the electromagnetic detector and the hadron calorimeter allows us to measure the axis of jets with an angular resolution of about $2.5^{\circ}$, and to measure the total energy of hadronic events from $Z^{0}$ decay with a resolution of $10 \%$ [7]. The muon detector consists of three layers of precise drift chambers. A detailed description of each detector subsystem, and its performance, is given in ref. [8].

For the present analysis, we use the data collected in the following ranges of polar angles:

- for the central chamber, $41^{\circ}<\theta<139^{\circ}$,

- for the electromagnetic calorimeter, $42^{\circ}<\theta<138^{\circ}$,

- for the hadron calorimeter, $5^{\circ}<\theta<175^{\circ}$,

- for the muon chambers, $36^{\circ}<\theta<144^{\circ}$.

\section{Event selection}

The primary trigger for hadronic events requires a total energy above $15 \mathrm{GeV}$ in the calorimeters, while the trigger for muons requires the signal of one of the 16 scintillation counter $\phi$ sectors to be in coincidence with a track in the muon chambers. The two triggers are in a logical OR with a charged track trigger and a scintillation counter multiplicity trigger. The combined trigger efficiency exceeds $99.9 \%$ for hadronic events, with or without leptons.

The selection of $\mathrm{e}^{+} \mathrm{e}^{-} \rightarrow$ hadrons events is based on the energy measured in the electromagnetic and hadronic calorimeters:

$0.6<E_{\mathrm{vis}} / \sqrt{s}<1.4$,

$\left|E_{\|}\right| / E_{\mathrm{vis}}<0.40, \quad E_{\perp} / E_{\mathrm{vis}}<0.50$,

$N_{\text {cluster }}>12$.

$E_{\text {vis }}$ is the total energy observed in the detector, $E_{\|}$ and $E_{\perp}$ are the parallel and transverse energy imbalances with respect to the beam direction. $N_{\text {cluster }}$ is the number of clusters, groups of neighbouring hits in the calorimeters with an energy exceeding $100 \mathrm{MeV}$. The algorithm normally reconstructs one cluster for each particle produced near the interaction point. The cut on the number of clusters rejects low multiplicity events $\left(\mathrm{e}^{+} \mathrm{e}^{-}, \mu^{+} \mu^{-}, \tau^{+} \tau^{-}\right)$. Applying the same cuts to simulated events, we estimate the acceptance to be $97 \%$.

Events of the type $Z^{0} \rightarrow b \bar{b}$ are selected identifying 
the muons or electrons coming from the semileptonic decay of the b quark.

Muons are identified and measured in the muon chamber system. A muon track must consist of track segments in at least two of three layers of the muon spectrometer. To reject the punch-through background, we require that the reconstructed track points to the interaction region.

Electrons are identified using the $\mathrm{BGO}$, the central tracking chamber and the hadron calorimeter. We require the lateral showed shape of the cluster in the BGO to be consistent with that of an electromagnetic shower. The energy in the hadron calorimeter behind this cluster must be small, and the centroid of the cluster must be matched to a track in the vertex chamber. The selection for both inclusive muon and electron events is described in detail in ref. [5].

Leptons coming from $b$ semi-leptonic decays have a large momentum and a large transverse momentum with respect to the $b$ quark direction. Therefore we require the muons and electrons to have a momentum greater than $4 \mathrm{GeV}$ and $3 \mathrm{GeV}$ respectively, and a transverse momentum with respect to the axis of the nearest jet greater than $1.5 \mathrm{GeV}$ for muons and 1 $\mathrm{GeV}$ for electrons. For this selection jets are reconstructed using the algorithm described in ref. [7 ]: the measured momentum of the lepton is excluded in the calculation of the jet direction. From 110000 hadronic events we select approximately 1800 muon and 1100 electron events.

Monte Carlo distributions were generated by the program JETSET 7.2 [9] with parton shower and string fragmentation. For the heavy quarks we use the Peterson fragmentation function [10] with parameters adjusted to match our measured inclusive muon data [5]. The generated events were passed through the L3 detector simulation [11] ${ }^{\# 1}$ which includes the effects of energy loss, multiple scattering, interactions and decay sin the detector materials and beam pipe. From Monte Carlo studies we estimate the $b$ purity to be $86 \%$ for the muon sample and $88 \%$ for the electron sample. The error on these numbers is estimated to be $\pm 5 \%[5]$.

\#I GEANT Version 3.13 (September 1989). Hadronic interactions are simulated using the GHEISHA program, see ref. [12].

\section{Determination of the 3 -jet rate}

In order to determine the number of jets in the selected samples of hadronic and inclusive lepton events, we use the JADE algorithm [12]. For each pair of "particles" (calorimetric clusters and muons) $i$ and $j$ we calculate the scaled invariant mass squared $y_{i j}=2 E_{i} E_{j} / E_{\text {vis }}^{2} \cdot\left(1-\cos \theta_{i j}\right)$, where $E_{i}$ and $E_{j}$ are the particle energies and $\theta_{i j}$ is the angle between particles $i$ and $j$. The pair for which $y_{i j}$ is smallest is replaced by a pseudoparticle $k$ with four-momentum $p_{k}=p_{i}+$ $p_{j}$. This procedure is repeated until all $y_{i j}$ exceed the jet resolution parameter $y_{\text {cut }}$. The remaining pseudoparticles are called jets.

We determine the 3 -jet rate

$f_{3}=\frac{N_{3-\text { jet }}}{N_{\text {tot }}}$

(where $N$ is the number of events) at nine equidistant values of $y_{\text {cut }}$ ranging from 0.02 to 0.10 , for the total hadronic sample

$f_{3}($ had $)=\frac{N_{3 \text {-jet }}(\text { had })}{N_{\text {tot }}(\text { had })}$

and for the inclusive muon and electron samples separately

$f_{3}(\mu)=\frac{N_{3 \text {-jet }}(\text { incl. muons })}{N_{\text {tot }}(\text { incl. muons })}$,

$f_{3}($ e $)=\frac{N_{3-\text { jet }}(\text { incl. electrons })}{N_{\text {tot }}(\text { incl. electrons })}$.

From these quantities we compute the ratios $f_{3}(\mu) /$ $f_{3}(\mathrm{had})$ and $f_{3}(\mathrm{e}) / f_{3}(\mathrm{had})$ for each value of $y_{\text {cut }}$.

In order to correct the two ratios we use the Monte Carlo JETSET 7.2. The corrected ratios are obtained by multiplying with a $y_{\text {cut }}$ dependent factor

$C=C_{\mathrm{d}} \cdot C_{\mathrm{h}} \cdot C_{\mathrm{b}}$.

The factors $C_{\mathrm{d}}$ take into account the detector effects and are evaluated from the parton shower Monte Carlo before and after including detector resolution and acceptance: they range from 0.90 to 1 . The factors $C_{\mathrm{h}}$ are due to fragmentation effects and are given by the ratio of $f_{3}(\mathrm{e}, \mu) / f_{3}$ (had) before and after hadronization: this gives a correction of typically $3 \%$. The numbers $C_{\mathbf{b}}$ have been evaluated using the first order QCD matrix element, including mass effects, assum- 
Table 1

Measured 3-jet rates for the inclusive muon and the inclusive electron samples for the nine different values of $y_{\mathrm{cut}}$. The ratios have been corrected for detector acceptance and resolution, for hadronization and bottom mass effect. The first error is statistical, the second is systematic.

\begin{tabular}{lll}
\hline$y_{\text {cut }}$ & $f_{3}(\mu) / f_{3}(\mathrm{had})$ & $f_{3}(\mathrm{e}) / f_{3}(\mathrm{had})$ \\
\hline 0.02 & $0.94 \pm 0.03 \pm 0.05$ & $0.94 \pm 0.04 \pm 0.05$ \\
0.03 & $0.98 \pm 0.04 \pm 0.05$ & $0.99 \pm 0.05 \pm 0.06$ \\
0.04 & $0.99 \pm 0.04 \pm 0.05$ & $1.00 \pm 0.05 \pm 0.06$ \\
0.05 & $0.99 \pm 0.04 \pm 0.05$ & $1.02 \pm 0.06 \pm 0.07$ \\
0.06 & $1.00 \pm 0.05 \pm 0.06$ & $1.02 \pm 0.07 \pm 0.07$ \\
0.07 & $0.97 \pm 0.05 \pm 0.06$ & $1.03 \pm 0.07 \pm 0.08$ \\
0.08 & $0.95 \pm 0.06 \pm 0.06$ & $1.02 \pm 0.08 \pm 0.08$ \\
0.09 & $0.92 \pm 0.06 \pm 0.07$ & $1.02 \pm 0.09 \pm 0.09$ \\
0.10 & $1.00 \pm 0.07 \pm 0.07$ & $1.04 \pm 0.09 \pm 0.09$ \\
\hline
\end{tabular}

ing a b quark mass of $5 \mathrm{GeV}$ [14]: the correction is around $+2 \%$.

The systematic uncertainty in the correction factor $C_{\mathrm{d}}$ has been evaluated by changing the energy response in different components of the calorimeter up to $10 \%$ in the Monte Carlo simulation. We find a systematic uncertainty of $3 \%$.

To study the systematic uncertainty in the hadronization correction, we vary the $\epsilon_{\mathrm{b}}$ parameter of the Peterson fragmentation function of the $b$ quark, and the fragmentation parameters $\sigma_{\mathrm{q}}$ and $b$, in a range compatible with our measured inclusive lepton spectra and event shape distributions $[5,15]$. We find a systematic uncertainty of $2 \%$ due to hadronization.

Table 1 shows the ratios $f_{3}(\mu) / f_{3}($ had $)$ and $f_{3}(\mathrm{e}) /$ $f_{3}(\mathrm{had})$ with the statistical and systematic errors, for the values of $y_{\text {cut }}$ considered. The systematic error also includes the Monte Carlo statistical error, which ranges from $2 \%$ to $5 \%$. There is good agreement between $\mu$ and e data, and the results are independent of the jet resolution parameter $y_{\text {cul }}$.

\section{Results and conclusions}

The two ratios for muons and electrons are finally combined in order to calculate the ratio between the 3-jet fraction for bottom events and for all other flavours. Assuming that there is no difference in the strong interaction for the quarks $u, d, s$ and $c$, we can write $\frac{f_{3}(\mathrm{e}+\mu)}{f_{3}(\mathrm{had})}=\frac{\left(1-p^{\mathrm{e}+\mu}\right) f_{3}^{\text {udsc }}+p^{\mathrm{e}+\mu_{3}^{\mathrm{b}}}}{\left(1-p^{\mathrm{had}}\right) f_{3}^{\text {udsc }}+p^{\mathrm{had}} f_{3}^{\mathrm{b}}}$,

where $p^{\mathrm{e}+\mu}$ is the combined b purity for the two samples of inclusive lepton events $((87 \pm 5) \%)$ and $p^{\text {had }}$ is the percentage of $b$ in hadronic events ( $(22 \pm$ $0.5) \%$ ).

In first order QCD, the 3-jet rate is proportional to $\alpha_{s}$ :

$f_{3}=A\left(y_{\text {cut }}\right) \alpha_{\mathrm{s}}$.

The function $A\left(y_{\text {cut }}\right)$ has been calculated in ref. [16]. From the ratio $f_{3}(\mathrm{~b}) / f_{3}$ (udsc) we can then determine the relative coupling strength for $b$ and the other quarks: fig. 1 shows the ratio $\alpha_{\mathrm{s}}(\mathrm{b}) / \alpha_{\mathrm{s}}(\mathrm{udsc})$ as a function of $y_{\text {cut }}$.

Taking into account second order corrections to the relation between the 3 -jet rate and $\alpha_{\mathrm{s}}$ [16], we find that the result changes by less than $1 \%$. For these corrections we used a value for $\alpha_{s}\left(M_{\mathrm{Z}}\right)=0.115$ as determined from our previous analysis [17], and we fix the renormalization scale $\mu^{2}$ to $y_{\text {cut }} s$.

For a value of $y_{\text {cut }}=0.05$, for which the 4-jet rate is small and the 3 -jet fraction is large $(29 \%)$, we measure $f_{3}(\mathrm{e}+\mu) / f_{3}(\mathrm{had})=1.00 \pm 0.03$ (stat. $) \pm$ 0.04 (syst.), from which we obtain

$\alpha_{\mathrm{s}}$ (b) $/ \alpha_{\mathrm{s}}$ (udsc) $=1.00 \pm 0.05$ (stat.) \pm 0.06 (syst.) ,

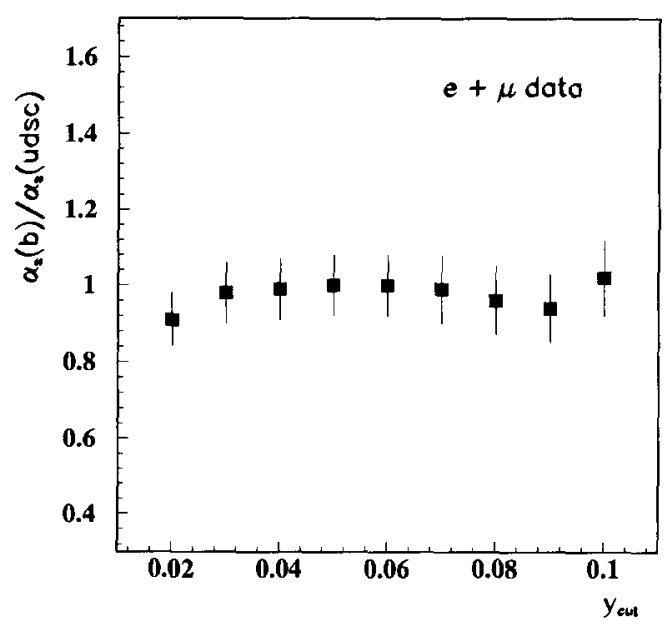

Fig. 1. Measured $\alpha_{s}(\mathrm{~b}) / \alpha_{s}(\mathrm{udsc})$ ratios, for the inclusive lepton sample (muons and electrons), as a function of the jet resolution parameter $y_{\text {cut }}$. The error includes the statistical and the systematic contributions. 
where the systematic error includes also the small contributions from the errors on the $p^{\mathrm{e}+\mu}$ and $p^{\text {had }}$ fractions. This result is consistent with the flavour independence predicted by QCD. The precision is significantly better than that previously achieved [2].

\section{Acknowledgement}

We wish to express our gratitude to the CERN accelerator divisions for the excellent performance of the LEP machine. We acknowledge the effort of all engineers and technicians who have participated in the construction and maintenance of this experiment.

\section{References}

[1] TASSO Collab., W. Braunschweig et al., Z. Phys. C 44 (1989) 365.

[2] TASSO Collab., W. Braunschweig et al., Z. Phys. C 42 (1989) 17.
[3] W. Kwong et al., Phys. Rev. D 37 (1988) 3210.

[4] T. Hebbeker, QCD at LEP/SLC, Aachen Report PITHA 91/ 11.

[5] L3 Collab, B. Adeva et al., Phys. Lett. B 261 (1991) 177; B 252 (1990) 713.

[6] L3 Collab, B. Adeva et al., L3 preprint \# 28 (February 1991 ).

[7] O. Adriani et al., Nucl. Instrum. Methods A 302 (1991) 53.

[8] L3 Collab., B. Adeva et al., Nucl. Instrum. Methods A 289 (1990) 35.

[9] T. Sjöstrand, Comput. Phys. Commun. 39 (1986) 347;

T. Sjöstrand and M. Bengtsson, Comput. Phys. Commun. 43 (1987) 367

[10] C. Peterson et al., Phys. Rev. D 27 (1983) 105.

[11] R. Brun et al., GEANT 3, report CERN DD/EE/84-1 (Revised) (September 1987).

[12] H. Fesefeldt, RWTH Aachen Report PITHA 85/02 (1985).

[13] JADE Collab., W. Bartel et al., Z. Phys. C 33 (1986) 23; JADE Collab., S. Bethke et al. Phys. Lett. B 213 (1988) 235.

[14] B.L. Ioffe, Phys. Lett. B 78 (1978) 277.

[15] L3 Collab., B. Adeva et al., Phys. Lett. B 257 (1991) 469.

[16] Z. Kunszt and P. Nason, Z Physics at LEP 1, CERN Report CERN-89-08, Vol. 1 (1989) p. 373.

[17] L3 Collab., B. Adeva et al., Phys. Lett. B 248 (1990) 464. 